\title{
ORIGINAL ARTICLE Spinal cord injury rehabilitation and mental health, SCReaM
}

\author{
N Warner, G Ikkos and A Gall
}

Aims: The aim of the study was to investigate whether people with a pre-existing mental health disorder (MHD) benefit from rehabilitation following a spinal cord injury $(\mathrm{SCl})$ and how their outcomes differ from those without a pre-existing MHD.

Methods: Rehabilitation outcomes of a cohort of patients with pre-existing MHD discharged from the London SCI Centre over a 6-year period were investigated. A retrospective matched case-control study design was used to compare the Spinal Cord Independence Measure III between those with an $\mathrm{SCl}$ and pre-existing MHD and those without and both compared with published expected outcomes. Results: The study found that, overall, those with MHD do benefit from SCI rehabilitation and that their outcomes do not significantly differ from those without MHD. Furthermore, the outcomes were favourable when compared with published expected outcomes.

Conclusion: Having a pre-existing MHD does not preclude patients with an $\mathrm{SCl}$ from benefiting from rehabilitation. These findings are an important basis on which to ensure equal access to rehabilitation for patients with a pre-existing MHD.

Spinal Cord (2017) 55, 307-313; doi:10.1038/sc.2016.107; published online 12 July 2016

\section{INTRODUCTION}

The World Health Organisation ${ }^{1}$ notes that over a third of people in most countries report problems at some time in their life, which meet the criteria for diagnosis of mental health disorder (MHD). ${ }^{2}$ Mental health problems have the highest burden of disease in the UK with approximately $46 \%$ of people with MHD having a co-morbid long-term physical health condition. ${ }^{3}$

Evidence shows that SCI rehabilitation programmes promote health, independence, participation and quality of life. ${ }^{4}$ However, there is evidence from the Kings Fund and Centre for Mental Health that health service providers do not do enough to accommodate mental health needs and do not support an integrated response to dual physical and mental health needs. ${ }^{3}$ The Cost of Co-Morbidities published in February 2012 highlights issues people with MHD and long-term physical health conditions face and suggests ways their care could be improved. It reports that patients with MHD not only carry more costs to the NHS but also that their condition contributes to poorer clinical outcomes and quality of life. ${ }^{3}$

Previous research looking specifically at MHD and spinal cord injury outcomes is limited but has, in general, found that outcomes do not greatly differ between those with pre-existing MHD and those without. ${ }^{5}$ These studies have been significantly limited for a number of reasons. First, they have had small sample sizes. Second, they have not used the current, internationally recognised activity outcome measure (Spinal Cord Independence Measure (SCIM)). Third, they have not used matched controls or compared outcomes with those empirically expected. ${ }^{5-8}$

We investigated rehabilitation outcomes of those discharged from the London SCI Centre (LSCIC) from April 2007 to April 2013 with a pre-existing MHD and compared outcomes with matched controls. A retrospective matched case-control study design was used to compare the Spinal Cord Independence Measure III (SCIM III) between those with an SCI and pre-existing MHD and those without, and compared with published expected outcomes; the multicentre study by Aidinoff et al. ${ }^{1}$ found that there is a negative correlation between the SCI level and 'discharge functional-score'. They conclude that the SCIM III scores from this study can be used as 'target' values for functional achievements of patients in rehabilitation with a complete SCI' and that the scores may also be used as a 'minimumtarget' for those with incomplete lesions. ${ }^{1,9}$

The study investigated the following:

1. Do people with a pre-existing MHD benefit from rehabilitation following SCI?

2. Do People with pre-existing MHD have poorer outcomes from SCI rehabilitation than those without a pre-existing MHD?

\section{MATERIALS AND METHODS}

This study was a retrospective matched case-control review: Between April 2007 and April 2013, 379 newly injured patients were discharged from LSCIC. Of these, 64 were identified as having a pre-existing MHD on admission; the MHD diagnosis (using ICD-10) was made or confirmed by a consultant psychiatrist based at the LSCIC. Patients with more than one psychiatric co-morbid diagnosis had their diagnoses ranked in order of priority, as laid out in the ICD-10. ${ }^{10}$ Patients' case notes were retrieved and used to supplement discharge summaries. International Spinal Cord Society (ISCOS) core data sets, ASIA impairment scale (AIS) and SCIM III scores and further details on MHD and aetiology of SCI were collected where possible. . $^{1,9,11,12}$

The 64 patients with pre-existing MHD (from here forth referred to as cases) were matched with controls (without MHD) based on gender, AIS and level of injury, ${ }^{2}$ age ( \pm 10 years) and year of injury ( \pm 2 year). ${ }^{11}$ For cervical and lumbar level injuries where functional ability is highly affected by variation in level, the ASIA charts were consulted to identify the best matches, based on motor levels 
and scores. A and B grade of injury (motor complete) were grouped together for matching and compared against motor incompletes. ${ }^{11}$

\section{Statistical analysis}

Matching of patients for gender, year of injury and AIS was evaluated using percentages. The matching of age was analysed using linear regression.

The parametric paired $t$-test was used to compare the SCIM outcomes between controls and cases. Subgroup analysis was conducted and the paired $t$-test was used to compare specific MHD groups, paraplegia with tetraplegia and motor complete versus motor incomplete. The two-way multivariate analysis of variance (MANOVA) was used to evaluate the significance of any differences between all MHD groups, based on the case and control pairs. This same analysis was also performed for paraplegic versus tetraplegic groups and the motor complete versus motor incomplete groups.

We used the parametric paired $t$-test to compare cases and controls observed discharge SCIM against the results of the study by Aidinoff et al., using their findings as an expected SCIM for complete injury and minimum expected-SCIM for incomplete injuries. ${ }^{1}$ Level of injury was used as the dependent variable.

\section{RESULTS}

\section{Matching}

Gender, age and year of injury. There was a $100 \%$ gender match between cases and controls. The gender ratio was $66: 34$ male to female. We aimed for a difference of less than 10 years in age between cases and controls, and this was achieved in 66\%. The linear relationship between case and control ages is shown in Figure 1. We successfully matched $81 \%$ of patients by year of injury $(<2 \pm$ years).

ASIA Impairment Scale. We attempted to exactly match cases to controls by level and completeness of injury. Degree of match for the various levels can be seen in Table 1; this has been broken down into upper cervical (c1-3), lower cervical (c4-8), thoracic and lumbar level

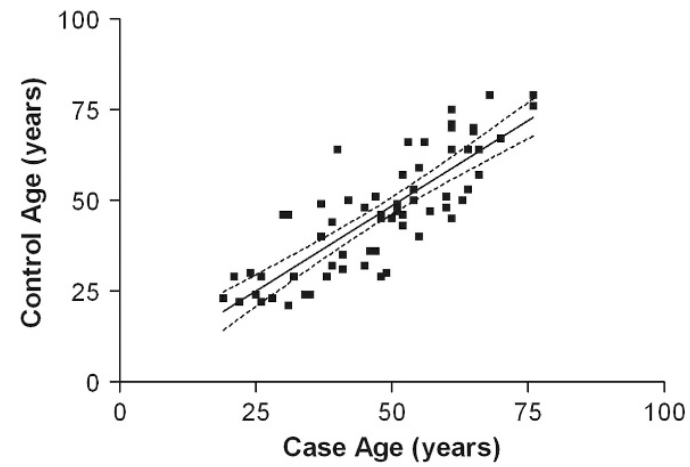

Figure 1 Scatter plot showing matching of case versus control age with linear regression $\left(y=0.93 x-1.8, r^{2}=0.68\right)$. (as per De Vivo et al. ${ }^{13}$ ). Degree of match by AIS grade is seen in Table 2, and exact matching of grade was achieved in $87.5 \%$.

\section{Mental health disorder diagnoses and patient demographics}

Table 3 shows the patient demographics. The aetiology of SCI for cases and controls is shown in Figure 2. Overall, falls was the most common mode of injury for both cases and controls, although the proportion of falls (including jumps) among cases (69\%) was higher compared with controls (44\%). ${ }^{12}$ From the available data, there were $19 \%$ confirmed suicide attempts among cases versus zero among controls.

Table 4 shows the frequency of different MHD diagnoses identified for both primary and psychiatric co-morbid MHD diagnoses in the study sample. Patients with more than one psychiatric co-morbid diagnosis had their diagnoses ranked by the ICD-10 diagnosis hierarchy. ${ }^{10}$ Of the 64 cases, 37 had 1 MHD, 16 had 2 MHDs, 9 had 3 MHDs, and 2 had 4 or more MHDs.

Injury to admission time, length of rehabilitation stay, total hospital stay and associated injuries. There was no significant difference between cases and controls for injury to admission time, length of stay (LOS, length of rehabilitation stay and total hospital stay) (Table 3). The large standard deviations observed are likely to reflect variations in length of stay for the different injury types, as these remain large even when outliers have been removed.

Cases with MHD tended to have more associated injuries, although no significant difference was found $\left(X^{2}\right.$ test, $\left.P=0.127\right)$ (Table 3$)$.

Discharge destination. ISCOS core data sets were used to categorise patients' discharge destinations. ${ }^{12}$ Differences were observed in discharge destination between cases and controls. This is illustrated in Figure 3. In the control group, $95 \%$ were discharged to a private residence whereas only $41 \%$ of cases were discharged to a private residence and $30 \%$ to a nursing home. A $X^{2}$ test confirmed a significant difference between proportion of cases and proportion of controls discharged to a nursing home $(P \leqslant 0.001)$.

Table 2 Degree of match by AIS grade, variation per grade and percentage of exact match

\begin{tabular}{lccc}
\hline & Matched by identical grade & Unmatched by 1 grade & Exact match (\%) \\
\hline AIS A & 24 & $3(3 \times \mathrm{A}-\mathrm{B})$ & 89 \\
AIS B & 3 & $2(1 \times \mathrm{B}-\mathrm{A}, 1 \times \mathrm{B}-\mathrm{C})$ & 60 \\
AIS C & 3 & $3(1 \times \mathrm{C}-\mathrm{B}, 2 \times \mathrm{C}-\mathrm{D})$ & 50 \\
AIS D & 26 & 0 & 100 \\
\hline
\end{tabular}

Table 1 Matching of levels as per De Vivo et al., ${ }^{13}$ variation per level and percentage of exact match

\begin{tabular}{lcccc}
\hline $\begin{array}{l}\text { Level of injury } \\
\text { subcategories }\end{array}$ & $\begin{array}{c}\text { Number of patients } \\
\text { exactly matched by level }\end{array}$ & $\begin{array}{c}\text { Number of patients } \\
\text { match } \pm \text { l level }\end{array}$ & $\begin{array}{c}\text { Number of patients } \\
\text { match } \pm \text { 2 levels }\end{array}$ & $\begin{array}{c}\text { Number of patients } \\
\text { match } \pm 3 \text { levels }\end{array}$ \\
\hline C1-C4 A, B, C & 8 & 2 & 0 & 0 \\
C1-C4 D & 10 & 4 & 0 & 0 \\
C5-C8 A, B, C & 2 & 1 & 0 & 0 \\
C5-8 D & 0 & 2 & 1 & 0 \\
T1-S5 A, B, C & 2 & 8 & 8 & 0 \\
T1-S5 D & 4 & 3 & 0 & 0 \\
\hline
\end{tabular}


Table 3 Demographics of injury for cases and controls including age, gender, type of injury (level and completeness), associated injuries and LOS (with outliers removed) ${ }^{12}$

\begin{tabular}{lccc}
\hline & Cases & Controls & P-value \\
\hline$N$ & 64 & 64 & \\
Age, mean \pm s.d. (median, range), & 48 s.d. 14 & 47 s.d. 16 & \\
years & $(50,19-76)$ & $(46,21-79)$ & \\
Males:females & $42: 22$ & $42: 22$ & \\
Tetraplegia & 31 & 31 & \\
Complete:incomplete & $9: 22$ & $7: 24$ & \\
Paraplegia & 33 & 33 & \\
Complete:incomplete & $18: 15$ & $18: 15$ & \\
Injury to admission time, mean \pm s.d. & 55 s.d. 51 (45) & 37 s.d. 41 (19) & 0.806 \\
(median), days & & & \\
Rehabilitation LOS, mean \pm s.d. & 111 s.d. 48 & 116 s.d. 50 & 0.968 \\
(median), days & $(105)$ & $(107)$ & \\
Total hospital LOS, mean \pm s.d. & 160 s.d. 63 & 148 s.d. 66 & 0.060 \\
(median), days & $(146)$ & $(122)$ & \\
Associated injuries & $12 / 64$ (19\%) & $6 / 64$ (9\%) & 0.127 \\
\hline
\end{tabular}

Abbreviations: LOS, length of stay.

\section{Outcomes}

Admission SCIM. The admission SCIM was used to ensure an equal baseline functional level between cases and controls. No significant difference was found between the admission SCIMs of cases and controls (mean difference of 2 s.d. 24, $P$-value of $0.566, r=0.05$ ) (Figure 4).

Discharge SCIM. The overall mean discharge SCIM was 51 for cases and 54 for controls.

No significant difference was found between discharge SCIM of cases and controls (mean difference -4 s.d. 26, $P$-value $=0.258$, $r=0.07$ ) (Figure 4).

SCIM gains for cases. A significant improvement in SCIM was observed in all cases between admission and discharge, with a mean difference of 31 s.d. $31(P \leqslant 0.001, r=0.58)$ (Figure 4).

SCIM gains between groups. No significant difference was found between SCIM gains of cases and controls (mean -2 s.d. 26, $P$-value $=0.555, r=0.05)($ Figure 4$)$.

Subgroup analysis of discharge SCIM. Paired t-test analysis showed that those patients with motor complete injuries, paraplegia, schizophrenia and non-affective psychosis and those with a co-morbid diagnosis of anxiety, stress-related and somatoform disorder had significantly worse outcomes (Table 4).

However, the two-way MANOVA showed no significant difference between MHD diagnosis subgroups $(P=0.733)$, no significant difference between paraplegic and tetraplegic groups $(P=0.408)$ and no significant difference between the motor complete and motor incomplete groups $(P=0.646)$. In spite of this, there was a marked difference in estimated marginal means between the cases and controls for the schizophrenia, co-morbid anxiety, self-harm, motor complete and paraplegic subgroups (Figure 5). Although there was no significant difference between cases and controls in the self-harm group using the $t$-test analysis, there was a moderate effect size significance. This suggests that the observed sample size may have been too small to reach statistical significance.

Observed versus expected SCIM. Among those with complete injuries in our control population, no significant difference was found between

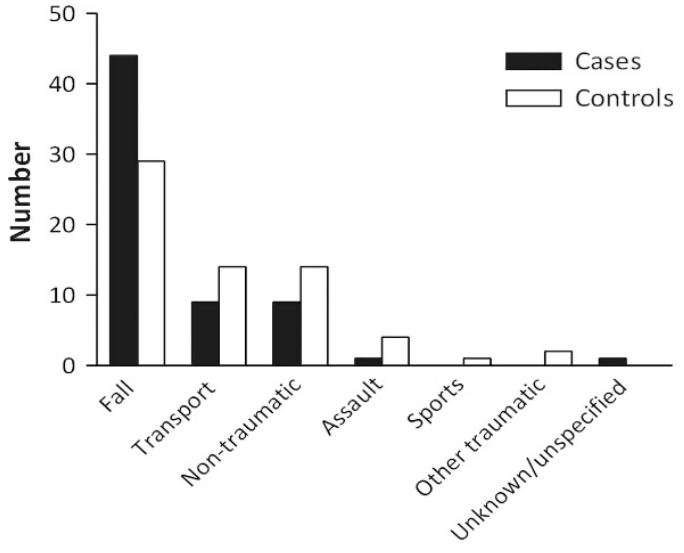

Figure 2 Graph showing mode of injury for cases and controls (according to ISCOS core data collection). ${ }^{12}$

observed and expected discharge SCIMs (mean difference 0.6 s.d. 12, $P$-value $=0.812, r=0.22)\left(\right.$ based on the findings from Aidinoff $e t$ al..$\left.^{1}\right)$

The majority of cases with complete injuries achieved at least the expected-SCIM outcomes (overall mean difference between observed and expected SCIM $=-4$ s.d. $11, P$-value $=0.126, r=0.08$ ). However, there was a significant negative difference found between the observed and expected discharge SCIM (median) for the complete paraplegics (mean difference of -7.0 s.d. $10, P$-value $=0.010, r=0.41){ }^{1}$

As expected, there was a positive significant difference between observed and expected discharge SCIM for the incomplete injuries (mean difference 19 s.d. 37, $P$-value $=0.004, r=0.32$ ), as findings of Aidinoff et al. ${ }^{1}$ were used as minimum-target values. ${ }^{1}$

\section{DISCUSSION}

This retrospective case-control study compared rehabilitation outcomes in all patients with a pre-existing MHD discharged from the LSCIC between 2007 and 2013, with matched controls without MHD. The prevalence of pre-existing MHD among patients in this sample was substantially larger than previous research has indicated. ${ }^{5}$ This may be related to the population coming from the London area, given previous evidence of a link between urbanicity and MHD rates and morbidity. ${ }^{14,15}$ Alternatively, it may be in other settings, MHD is underrecognised or people with MHD are not being admitted for rehabilitation. Studies looking at MHD prevalence in those who have sustained their SCI through a suicide attempt have higher prevalence figures. $8,16-18$

The first question our study investigated was do those with MHD benefit from rehabilitation following an SCI? We found that overall they do benefit as there was a significant positive difference between admission SCIM and discharge SCIM (SCIM gain), with a large effect size. We also compared SCIM gains between the cases and controls and found no significant difference with equivalent admission SCIMs.

Discharge SCIM III was used as our final objective measurement of functional ability at discharge. Overall, there was no significant difference for the discharge SCIM between the cases and controls, which answers our second question-do people with MHD have poorer outcomes from SCI rehabilitation than those without MHD? This supports the research by Kennedy et al. ${ }^{5}$ in 2009 who demonstrated improving scores of the Needs Assessment Checklist in patients with MHD.

The two-way MANOVA found overall no significant difference between the MHD subgroups, suggesting similar rehabilitation outcomes between the various MHD groups. However, when the 
Table 4 SCIM results for the different categories of injury type and MHD (primary and co-morbid psychiatric diagnosis as per ICD-10 (codes shown above ${ }^{10}$ )

\begin{tabular}{|c|c|c|c|c|c|}
\hline Subgroups: injury types and MHD & Number of cases & $\begin{array}{c}\text { Cases mean admission SCIM } \\
+ \text { s.d. }\end{array}$ & $\begin{array}{c}\text { Cases mean discharge SCIM } \\
+ \text { s.d. }\end{array}$ & $\begin{array}{l}\text { Controls mean discharge } \\
\qquad S C I M+s . d .\end{array}$ & P-value (effect size) \\
\hline $\begin{array}{l}\text { Motor complete } \\
\text { ( } A+B \text { grade injuries) }\end{array}$ & 32 & 17 s.d. 11 & 42 s.d. 23 & 49 s.d. 23 & $P=0.043(r=0.15)$ \\
\hline $\begin{array}{l}\text { Motor incomplete } \\
\text { (C+D grade injuries) }\end{array}$ & 32 & 24 s.d. 18 & 60 s.d. 26 & 61 s.d. 27 & $P=0.781(r=0.02)$ \\
\hline Paraplegics & 33 & 23 s.d. 11 & 60 s.d. 17 & 67 s.d. 15 & $P=0.042(r=0.21)$ \\
\hline \multicolumn{6}{|l|}{ Major affective disorder F30-F39 } \\
\hline Primary & 20 & 24 s.d. 20 & 45 s.d. 27 & 41 s.d. 23 & $P=0.608(r=0.08)$ \\
\hline Co-morbid & 25 & 22 s.d. 18 & 48 s.d. 27 & 52 s.d. 27 & $P=0.489(r=0.07)$ \\
\hline \multicolumn{6}{|l|}{ Alcohol-related disorder F10 } \\
\hline Primary & 3 & 27 s.d. 17 & 51 s.d. 33 & 58 s.d. 34 & $P=0.135(r=0.10)$ \\
\hline Co-morbid & 6 & 18 s.d. 15 & 49 s.d. 26 & 50 s.d. 28 & $P=0.635(r=0.02)$ \\
\hline \multicolumn{6}{|c|}{ Anxiety, stress-related and somatoform disorders F40-F48 } \\
\hline Primary & 6 & 28 s.d. 13 & 54 s.d. 22 & 60 s.d. 21 & $P=0.133(r=0.14)$ \\
\hline Co-morbid & 14 & 24 s.d. 11 & 53 s.d. 20 & 62 s.d. 17 & $P=0.028(r=0.24)$ \\
\hline \multicolumn{6}{|c|}{ Schizophrenia and non-affective psychosis F20-F29 } \\
\hline Primary & 9 & 17 s.d. 11 & 48 s.d. 23 & 67 s.d. 15 & $P=0.032(r=0.44)$ \\
\hline Co-morbid & 9 & 17 s.d. 11 & 48 s.d. 23 & 67 s.d. 15 & $P=0.032(r=0.44)$ \\
\hline \multicolumn{6}{|l|}{ Self-harm X71-X83 } \\
\hline Primary & 6 & 24 s.d. 11 & 55 s.d. 8 & 69 s.d. 20 & $P=0.262(r=0.42)$ \\
\hline
\end{tabular}

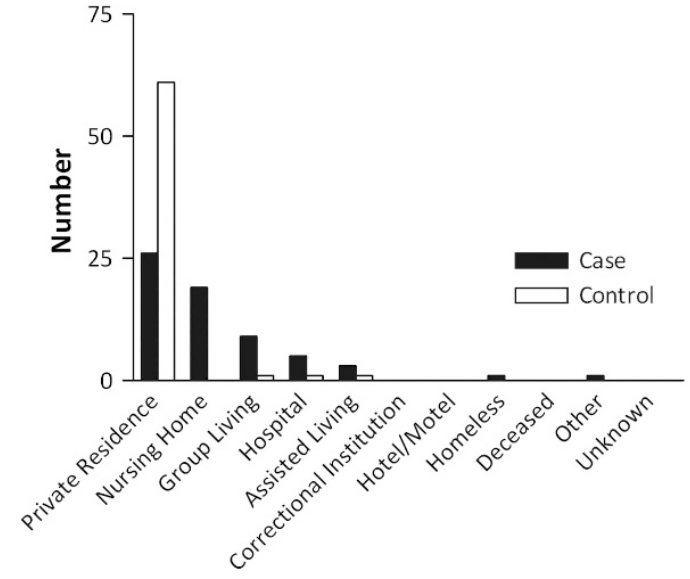

Figure 3 Graph showing discharge destination for cases and controls according to ISCOS core data categories. ${ }^{12}$

marginal means of the cases are compared with their controls, there is a marked difference for the co-morbid anxiety group, schizophrenia and self-harm groups as seen in Figure 5. This was confirmed by the $t$-tests that showed significantly different discharge SCIMs between the

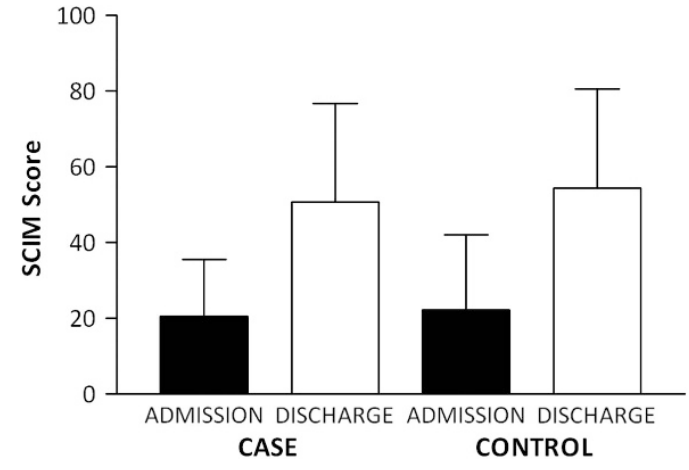

Figure 4 Graph showing admission and discharge SCIMs for all cases and controls with standard error bars.

cases and controls of those with schizophrenia or non-affective disorder and those with anxiety as a co-morbid psychiatric diagnosis.

These findings support the small study small study of Liang et al. ${ }^{7}$ in a Japanese rehabilitation centre in 1996 that concluded that psychotic symptoms hindered rehabilitation in their schizophrenic population. Self-harm, which includes suicide attempts, has also previously been shown to predict poorer outcomes in rehabilitation. ${ }^{16-18}$ 
Estimated Marginal Means of all MHD subgroups (including co-morbid
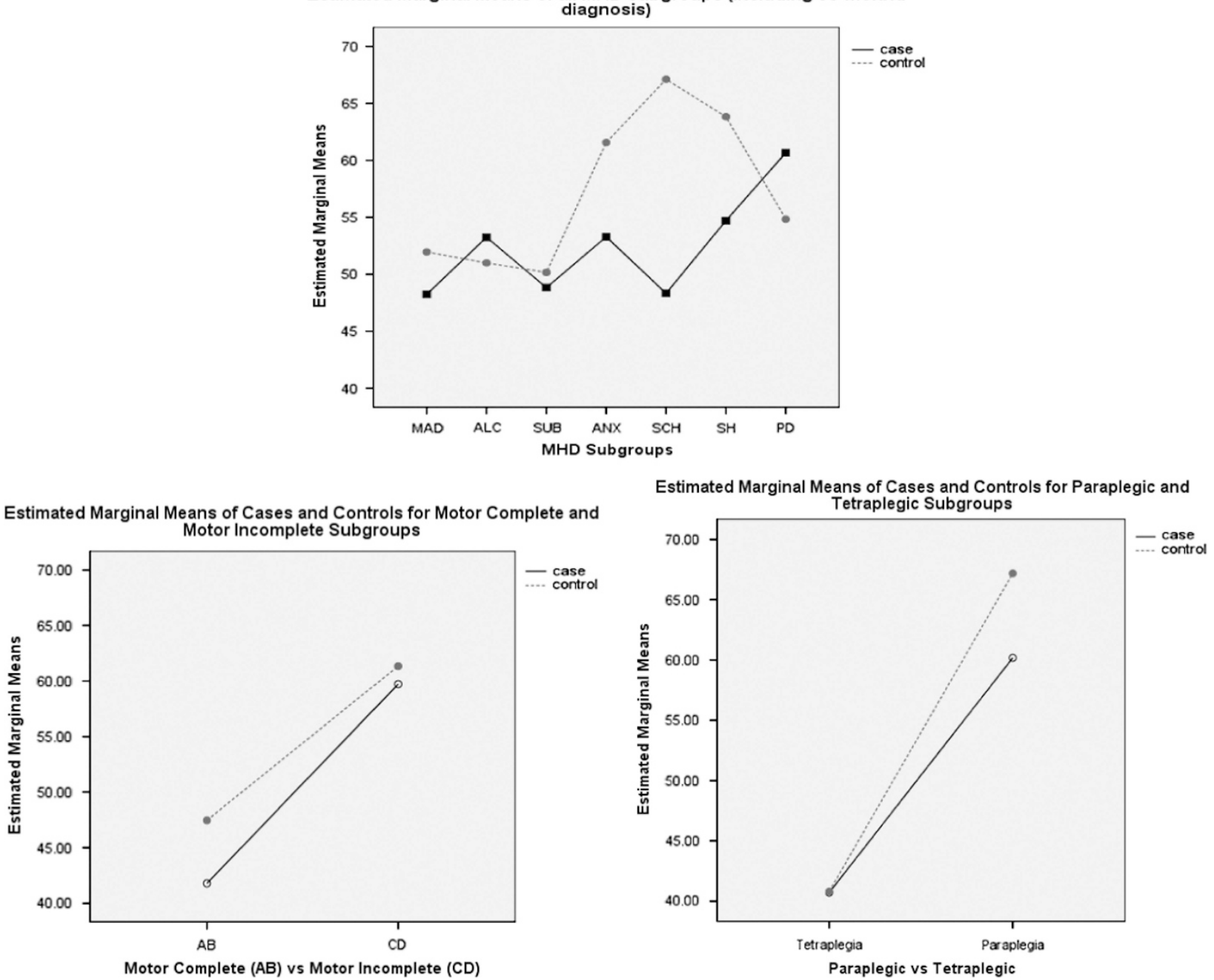

Figure 5 Graphs showing estimated marginal means between the cases and controls for MHD subgroups and for motor complete versus motor incomplete and paraplegic versus tetraplegic groups from the two-way MANOVA analysis.

Our results also indicate that anxiety as a psychiatric co-morbidity affects rehabilitation outcome. We hypothesise that the combination of lack of confidence, arousal and 'catastrophising' behaviours, as well as the challenges of relearning tasks may overwhelm patients with anxiety disorder, leading to worse rehabilitation outcomes. The fact that anxiety, stress-related and somatoform disorders as a primary diagnosis did not affect outcomes may relate to the ICD-10 hierachy of diagnoses ( 6 had a primary diagnosis compared to 14 overall) or that more generally anxiety disorder is poorly managed as a psychiatric co-morbid condition. ${ }^{10}$

The two-way MANOVA was also used to compare outcomes between the paraplegic and tetraplegic groups and the motor complete versus motor incomplete groups; although no significant difference was found, a difference was observed in the estimated marginal means (Figure 5). The paired $t$-test analysis also showed that those cases with motor complete injuries and those with paraplegia had significantly poorer discharge SCIMs compared with their controls and compared with 'expected' SCIM scores of Aidinoff et al., with a moderate effect size.

Other factors that could have potentially affected rehabilitation outcomes for the cases with complete injuries and those with paraplegia were analysed for trends; a high proportion, compared with the rest of the cases, had more than one associated injury, had their injury as a result of a suicide attempt and had co-morbid psychiatric diagnoses. However, sample sizes were too small to reach statistical significance.

Discharge destination is determined by a combination of patient choice (usually home), accessibility and ongoing needs. ${ }^{19}$ In the current study, there was a significant difference in discharge destination between the cases and controls; almost all controls were discharged to private residence compared with just under half of cases (Figure 3).

In the study by Anderson and Allan ${ }^{8}$ based in Scotland where outcomes of those injured by deliberate self-harm were studied, $80 \%$ returned to a private home. Stanford et al. ${ }^{16}$ looked at long-term outcomes in SCI related to suicide attempt and also found a high percentage $(70 \%)$ returned home, much higher than our cases; however, their study was conducted in Australia where health and social care models differ from the UK. In Kennedy et al. ${ }^{\prime 18}$ study at Stoke Mandeville, UK, only $25.5 \%$ of their deliberate self-harm group discharged home.

Rehabilitation aims to reduce health, functional and psychological needs. Our results demonstrate similar functional ability, suggesting that it may be the mental health needs that determine discharge 
destination. Community physical and mental health are separate within NHS as is social care; therefore, new physical impairments mean patients are often seen as ineligible for secondary mental health services. ${ }^{20}$ This may help explain the difference in discharge destination observed in our study, reflecting on the perceived complexity of the need and/or the lack of resources available to meet these patients' needs in a home environment. Patients with additional MHD needs may benefit from more integrated support than can currently be provided at home with the current configuration of services in England.

\section{Limitations}

Although this study is the largest to date to look at rehabilitation outcomes in those with a pre-existing MHD, the sample size is still relatively small, and therefore results need to be interpreted with caution, especially when interpreting the subgroup analysis, which includes groups that are very small and heterogeneous.

Statistics on demographics had to be compared with US demographics, as currently there is no published UK data. ${ }^{21}$ Normal gender ratio in SCI statistics is 80 to $20 \%$; however, here we found a ratio of 66 to $34 \%$; this may be related to the fact that there is a higher prevalence of MHD in women compared with men. ${ }^{13,21}$ Generally, the patients were well-matched. However, because of the size of the cohort, this was not exact.

Confounding variables not accounted for in our study included medical co-morbidities, medical complications during rehabilitation, family and social support, employment, socioeconomic status, pre-injury living situation including accessibility of accommodation, level of education and associated injuries (not part of ISCOS core data), specifically limb fracture managed conservatively. ${ }^{12}$ These variables could have impacted on factors such as length of stay, discharge destination and ultimately impacted on the rehabilitation (SCIM) outcome.

Limitations relating to the observed measurements include retrospective MHD diagnosis, no recorded measure of MHD severity and inadequate information indicating the contribution of the MHD to the injurious event. In some, the mechanism of falls or a confirmed aetiology was not available. Notably, 'jumps' are included in the 'falls' category of ISCOS core data. The available data suggest a higher incidence of suicide attempts among cases. This could account for the higher proportion of falls in this group.

Furthermore, our study did not look at the subsections of SCIM or measure coping or adjustment. ${ }^{6,9}$ In addition, the observed difference between tetraplegic and paraplegic cases observed could reflect on the floor and ceiling effect of the SCIM III for upper cervical and lower level injuries, respectively. ${ }^{9}$

\section{Further study}

The new national UK database, with details of MHD, could facilitate a large, national, prospective, study to determine the robustness of the results. ${ }^{22}$ In addition, it will be useful in future research to measure severity of MHD, number of rehabilitation days lost to psychiatric illness, complication rates, 1:1 nursing need, whether the aetiology of injury is specifically related to the MHD, the relationship between selfharm history/suicide attempt with rehabilitation outcomes and how the other confounding factors previously mentioned may have a part in the findings.

For the groups that had poorer rehabilitation outcomes, further analysis of separate SCIM sections is required to see which areas these patients performed poorer in and to allow more detailed clinical recommendations to improve outcomes for such patients.
The LSCIC is fortunate to have a full-time integrated psychiatry service, as a number of the other SCICs across the UK do not have such a service, often relying on part-time or external services. It would be useful to perform the same study in a centre that does not have access to the same mental health resources and to understand the optimal skill mix and professional groups to deliver the most effective service.

It will be beneficial to further study and understand factors influencing discharge and discharge destination for this patient group and explore whether pre-injury living situation affects destination. It will also be interesting to study outcomes post discharge. Do these patients become more or less independent in the long term? What are the participation outcomes and quality of life both before and after discharge? Does their MHD lead to self-neglect in long term, causing secondary complications such as pressure sores, or do active psychotic symptoms prevent them from participating in activities of daily living (ADLs)? Do they go on to self-harm or complete suicide or request euthanasia abroad?

Our results show that those with anxiety disorder as a co-morbid psychiatric disorder had poorer outcomes; further research is needed to understand this and to look at effective interventions that can be implemented to help neutralise the effect of anxiety disorder on the SCI rehabilitation programme.

\section{CONCLUSION}

In our experience, historically, there have been poor expectations of patients with a pre-existing MHD in SCI rehabilitation. In summary, our findings suggest that those with an MHD do benefit from SCI rehabilitation for all levels and grade of SCI and that generally the observed benefit is as great as for those without an MHD. In addition, the outcomes, we argue, compare favourably with published expected outcomes.

However, in those with pre-existing anxiety (as a co-morbid psychiatric diagnosis), schizophrenia, a history of self-harm or suicide attempt and for those with complete paraplegia, the observed benefit is not as great. Further work is required to understand this, as well as the difference in discharge destination observed.

Clinicians and commissioners should be aware that SCI rehabilitation is beneficial and work to ensure that patients with MHD have full access to these services.

\section{DATA ARCHIVING}

There were no data to deposit.

\section{CONFLICT OF INTEREST}

The authors declare no conflict of interest.

\section{ACKNOWLEDGEMENTS}

We thank Dr Jan Gawronski, Consultant Spinal Cord Injury Rehabilitation, LSCIC; Dr Susie Lingwood, Consultant Psychiatrist, LSCIC; Dr Susie Cro, Honary Lecturer in Stastics, UCL; Dr Sarah Knight, Clinical Scientist, LSCIC; Professor Malcolm Warner, University of Cambridge; and Professor Ricardo Peccei, Kings College London.

1 Aidinoff E, Front L, Itzkovich M, Bluvshtein V, Gelernter I, Hart J. Expected spinal cord independence measure, third version, scores for various neurological levels after complete spinal cord lesions. Spinal Cord 2011; 49: 893-896.

2 WHO International Consortium in Psychiatric Epidemiology. Cross-national comparisons of the prevalences and correlates of mental disorders. Bull World Health Organ 2000; 78: 413-426. 
3 The King's Fund and Centre of Mental Health. Long term conditions and mental health conditions. the cost of co-morbidities, 2012. Available at http://www.kingsfund.org.uk/ sites/files/kf/field/field_publication_file/long-term-conditions-mental-health-cost-comorbidities-naylor-feb12.pdf.

4 De Vivo MJ, Richard JS, Stover SL, Go BK. Spinal cord injury. Rehabilitation adds life to years. West J Med 1991; 154: 602-606.

5 Kennedy P, Sherlock O, Nandu N. Rehabilitation outcomes in people with pre-morbid MHDs following SCl. Spinal Cord 2009; 47: 290-294.

6 Dawson J, Shamley D, Jamous MA. A structured review of outcome measures used for the assessment of rehabilitation interventions for SCI. Spinal Cord 2008; 46: 768-780.

7 Liang HW, Wang YH, Wang TG, Tang FT, Lai JS, Lien IN. Clinical experience in rehabilitation of $\mathrm{SCl}$ associated with schizophrenia. Arch Phys Med Rehabil 1996; 77: 283-286.

8 Anderson J, Allan DB. Vertebral fracture secondary to suicide attempt: demographics and patient outcome in a Scottish spinal rehabilitation unit. J Spinal Cord Med 2011; 34: 380-387.

9 The Spinal Cord Independence Measure III (SCIM III). Available at http://www.scireproject.com/book/export/html/152.

10 World Health Organisation. ICD-10 Classifications of Mental and Behavioural Disorder: Clinical Descriptions and Diagnostic Guidelines. World Health Organisation: Geneva, 1992.

11 American Spinal Injury Association. International Standards for Neurological Classification of SCl. American Spinal Injury Association: Chicago, 2011.
12 The International Spinal Cord Society. Core data set. Available at http://iscos.org.uk/ sitefiles/PageFile_20_coredataset.pdf.

13 DeVivo M, Biering-Sørensen F, Charlifue S, Noonan V, Post M, Stripling T. International SCl Core Data Set. Spinal Cord 2006; 44: 535-540.

14 Paykel ES, Abbott R, Jenkins R, Brugha TS, Meltzer H. Urban-rural mental health differences in Great Britain: findings from the National Morbidity Survey. Int Rev Psychiatry 2003; 15: 97-107.

15 Penkallas AM, Kohler S. Urbanicity and mental health in Europe: a systematic review. Eur J Mental Health 2014; 9: 163-177.

16 Stanford RE, Soden R, Bartrop R, Mikk R, Taylor TKF. Spinal cord and related injuries after attempted suicide: psychiatric diagnosis and long-term follow-up. Spinal Cord 2007; 45: 437-443.

17 Biering-Sorensen MD, Pedersen F, Müller W. SCI due to suicide attempt. Paraplgia 1992; 30: 139-144.

18 Kennedy P, Rogers B, Speer S, Frankel H. Spinal cord injuries and attempted suicide: a retrospective review. Spinal Cord 1999; 37: 847-852.

19 Devivo MJ. Discharge disposition from model spinal cord injury care system rehabilitation programs. Arch Phys Med Rehabil 1999; 80: 785-790.

20 Darzi A. High quality care for all: NHS Next stage review. Final report. Department of Health, 2007. Available at http://webarchive.nationalarchives.gov.uk/20130107105 354/http:/www.dh.gov.uk/prod_consum_dh/groups/dh_digitalassets/@dh/@en/documents/digitalasset/dh_085828.pdf

21 The National SCI Statistical Center. SCI facts and figures at a glance, 2013. Available at https://www.nscisc.uab.edu/PublicDocuments/fact_Figures_docs/Facts\%202013.pdf.

22 National Spinal Cord Injury Databse, 2014. Available at http://www.nscisb.nhs.uk/. 